\title{
UV fixed-point structure of the three-dimensional Thirring model
}

\author{
Holger Gies: and Lukas Janssent \\ Theoretisch-Physikalisches Institut, Friedrich-Schiller-Universität Jena, Max-Wien-Platz 1, 07743 Jena, Germany
}

(Dated: September 26, 2018)

\begin{abstract}
We investigate the UV fixed-point structure of the three-dimensional Thirring model by means of the functional renormalization group (RG). We classify all possible 4-fermi interactions compatible with the present chiral and discrete symmetries and calculate the purely fermionic RG flow using a full basis of fermionic fourpoint functions in the point-like limit. Our results show that the UV complete (asymptotically safe) Thirring model lies in a two-dimensional coupling plane which reduces to the conventional Thirring coupling only in the strict large- $N_{\mathrm{f}}$ limit. In addition to the Thirring universality class which is characterized by one relevant direction (also at finite $N_{\mathrm{f}}$ ), two further interacting fixed points occur which may define new universality classes of second-order phase transitions also involving parity-broken phases. The $N_{\mathrm{f}}$-dependence of the Thirring fixed point sheds further light on the existence of an $N_{\mathrm{f}}$-controlled quantum phase transition above which chiral symmetry remains unbroken for arbitrary large coupling, even though a definite answer will require a direct computation of competing orders.
\end{abstract}

\section{INTRODUCTION}

Three-dimensional relativistic fermionic systems have extensively been investigated in the literature in a variety of scenarios. On the one hand, they are per se interesting field theories with unconventional features, on the other hand they allow for fascinating applications to condensed-matter systems. In particular, three-dimensional quantum electrodynamics $\left(\mathrm{QED}_{3}\right)$ or the Thirring model [1] are actively discussed, e.g., as effective theories describing different regions of the phase diagram of high- $T_{\mathrm{c}}$ cuprate superconductors [2-4] and, recently, the electronic properties of graphene [5-9]. Especially graphene since its first synthesis in 2004 [10] is being lively discussed in the rapidly growing literature on this subject, also because it offers the appealing opportunity of a comparatively simple experimental realization of some up to now unobserved quantum relativistic phenomena, such as the Klein paradox [11] or Zitterbewegung [12]; for reviews, see [13, 14]. More exotically, some features of $3 d$ relativistic fermion systems can serve as toy models, e.g., for the standard model of particle physics [15], or a possible candidate for a "Theory of Everything" [16].

However, $\mathrm{QED}_{3}$ [17-23] and the three-dimensional Thirring model [24-31] are likewise intrinsically interesting, in particular because the ground states in these theories are expected to show a sensitivity to the number of fermion flavors $N_{\mathrm{f}}$. Several approximate solutions of the Dyson-Schwinger equations (DSE) predict that chiral symmetry breaking $(\chi \mathrm{SB})$ is prohibited once $N_{\mathrm{f}}$ is larger than a critical value $N_{\mathrm{f}}^{\mathrm{cr}}$ [26, 28, 29]. A similar quantum critical phenomenon has also been identified in many-flavor $\mathrm{QCD}_{4}$ [32, 33], being currently under intense investigation also because of its potential relevance for technicolor scenarios [34] and its implications for the thermal phase boundary [35].

The search for the quantum critical point in the Thirring model has so far been rather challenging: different DSE approaches, e.g., have yielded values between $N_{\mathrm{f}}^{\mathrm{cr}} \simeq 3.24$ [26]

\footnotetext{
*holger.gies@uni-jena.de

† lukas.janssen@uni-jena.de
}

and $N_{\mathrm{f}}^{\text {cr }}=\infty$ [27]. Recent extensive lattice simulations now point to $N_{\mathrm{f}}^{\mathrm{cr}} \simeq 6.6$ [31]. For physical graphene and cuprates, the number of flavors is $N_{\mathrm{f}}=2$, such that the true value of $N_{\mathrm{f}}^{\mathrm{cr}}$ may be of profound importance for physical effects corresponding to chiral symmetry breaking in the effective theories. In fact, a dynamically induced mass gap in the band structure of graphene, corresponding to a semimetal-Mott insulator quantum phase transition, could provide extraordinary applications for graphene-based electronics, offering a possible new candidate material to take over from Si-based technology [13].

In this work, we take a more fundamental viewpoint in order to investigate the amount of universality that can be attached to a possible value for $N_{\mathrm{f}}^{\mathrm{cr}}$. Naively, a universal answer for $N_{\mathrm{f}}^{\mathrm{cr}}$ may not be apparent as $3 d$ fermion models are perturbatively nonrenormalizable. Nevertheless, renormalizability of the $3 d$ Thirring model to any order in a large- $N_{\mathrm{f}}$ expansion has been shown in [24-27], with a diagrammar being very similar to $\mathrm{QED}_{3}$. This has been taken as an indication that the Thirring model can indeed by defined nonperturbatively in $3 d$, providing the same amount of universality as any perturbatively renormalizable theory.

Universality and, more profoundly, UV completeness can, in fact, be analyzed within Weinberg's scenario of asymptotic safety [36, 37], in which a UV complete infinite-cutoff limit can be taken at a potentially non-Gaußian fixed point. The resulting theories are predictive and exhibit universality, if the number of RG relevant directions at the fixed point are finite. In this work, we re-examine renormalizability of the $3 d$ Thirring model from this viewpoint by means of the functional renormalization group formulated in terms of the Wetterich equation [38]. Using a purely fermionic RG as a first step, the UV fixed-point structure can indeed be mapped out, on the one hand confirming the large- $N_{\mathrm{f}}$ results, but also revealing interesting deviations from the large- $N_{\mathrm{f}}$ asymptotics. For instance, we can identify a non-Gaußian UV fixed point that defines a "Thirring universality class" which, however, corresponds to a pure Thirring coupling only in the strict large- $N_{\mathrm{f}}$ limit. In addition, we find further fixed points which may be associated with phase transitions (and corresponding new universality classes), e.g., towards a parity broken phase. 
This paper is organized as follows. In Sec. II we analyze the discrete and continuous symmetries of the classical theory. We classify the different possible mass terms as well as all possible fermionic interaction channels in the point-like four-fermion limit. With the aid of the functional RG, we investigate the purely fermionic RG flow in Sec. III followed by an analysis of the fixed-point structure of the given class of models for varying number of fermion flavors in Sec. IV In Sec. V], we classify the resulting RG trajectories to the longrange physics, pointing to the existence of various phases with dynamical mass generation, and obtain a first glance at a possible mechanism for the formation of a critical flavor number, above which chiral symmetry breaking disappears. We give our conclusions in Sec.VI

\section{SYMMETRIES OF THE THIRRING MODEL}

The Lagrangian for the massless Thirring model in three Euclidean space-time dimensions is

$$
\mathcal{L}=\bar{\psi}^{a} \mathrm{i} \not \partial \psi^{a}+\frac{\bar{g}}{2 N_{\mathrm{f}}}\left(\bar{\psi}^{a} \gamma_{\mu} \psi^{a}\right)^{2}
$$

satisfying Osterwalder-Schrader positivity 11 The index $a$ runs over $N_{\mathrm{f}}$ distinct fermion flavors. In three dimensions, we could use $2 \times 2$ matrices (e.g., the Pauli matrices) as an irreducible representation of the Euclidean Dirac algebra

$$
\left\{\gamma_{\mu}, \gamma_{v}\right\}=2 \delta_{\mu \nu}
$$

but this representation does not permit a chiral symmetry. We therefore work exclusively with a $4 \times 4$ reducible representation of the Dirac algebra; an explicit representation is given by

$$
\gamma_{\mu}=\left(\begin{array}{cc}
0 & -\mathrm{i} \sigma_{\mu} \\
\mathrm{i} \sigma_{\mu} & 0
\end{array}\right), \quad \mu=1,2,3,
$$

with $\left\{\sigma_{1}, \sigma_{2}, \sigma_{3}\right\}$ being the $2 \times 2$ Pauli matrices. Thus $\psi^{a}$ represents a four-component Dirac spinor. Now there are two further $4 \times 4$ matrices which anticommute with all $\gamma_{\mu}$ as well as with each other,

$$
\gamma_{4}=\left(\begin{array}{ll}
0 & \mathbb{1} \\
\mathbb{1} & 0
\end{array}\right) \quad \text { and } \quad \gamma_{5}=\gamma_{1} \gamma_{2} \gamma_{3} \gamma_{4}=\left(\begin{array}{cc}
\mathbb{1} & 0 \\
0 & -\mathbb{1}
\end{array}\right)
$$

The massless Lagrangian (1) then is invariant under the axial transformations

$$
\begin{array}{lll}
\mathrm{U}_{\gamma_{4}}(1): & \psi \mapsto \mathrm{e}^{\mathrm{i} \alpha \gamma_{4}} \psi, & \bar{\psi} \mapsto \bar{\psi} \mathrm{e}^{\mathrm{i} \alpha \gamma_{4}}, \\
\mathrm{U}_{\gamma_{5}}(1): & \psi \mapsto \mathrm{e}^{\mathrm{i} \beta \gamma_{5}} \psi, & \bar{\psi} \mapsto \bar{\psi} \mathrm{e}^{\mathrm{i} \beta \gamma_{5}},
\end{array}
$$

\footnotetext{
${ }^{1}$ For Osterwalder-Schrader positivity [39], we require invariance of the action under (generalized) complex conjugation defined by $\psi^{\dagger}:=\mathrm{i} \bar{\psi} \gamma_{3}$ together with reflection of the (euclidean) time coordinate (which we choose to be $x_{3}$ ). For a detailed discussion of our chiral conventions, see e.g., Ref. [40].
}

as well as the vector transformations

$$
\begin{aligned}
\mathrm{U}_{\mathbb{1}}(1): & \psi \mapsto \mathrm{e}^{\mathrm{i} \vartheta} \psi, & \bar{\psi} \mapsto \bar{\psi} \mathrm{e}^{-\mathrm{i} \vartheta}, \\
\mathrm{U}_{\gamma_{45}}(1): & \psi \mapsto \mathrm{e}^{\mathrm{i} \varphi \gamma_{45}} \psi, & \bar{\psi} \mapsto \bar{\psi} \mathrm{e}^{-\mathrm{i} \varphi \gamma_{45}},
\end{aligned}
$$

with $\gamma_{45}:=\mathrm{i} \gamma_{4} \gamma_{5}$. For each flavor $a=1, \ldots, N_{\mathrm{f}}$, the theory thus has a global U(2) symmetry with the Hermitian generators $\tau_{j}, j=1, \ldots, 4$,

$$
\tau_{j}=\mathbb{1}, \gamma_{4}, \gamma_{5}, \gamma_{45}
$$

This symmetry transformations together with flavor rotations form a larger symmetry of the classical massless Lagrangian, corresponding to the group $\mathrm{U}\left(2 N_{\mathrm{f}}\right)$ with the $\left(2 N_{\mathrm{f}}\right)^{2}$ generators

$$
\lambda_{i} \otimes \tau_{j}, \quad i=1, \ldots, N_{\mathrm{f}}^{2}, \quad j=1, \ldots, 4 .
$$

Here, $\left\{\lambda_{1}, \ldots, \lambda_{N_{\mathrm{f}}{ }^{2}-1}\right\}$ are the generalized $N_{\mathrm{f}} \times N_{\mathrm{f}}$ Gell-Mann matrices, and $\lambda_{N_{\mathrm{f}}^{2}}:=\mathbb{1}_{N_{\mathrm{f}}}$ is the identity. In other words, combining the $N_{\mathrm{f}}$ four-component spinors $\psi^{a}=\left(\psi_{\mathrm{L}}^{a}, \psi_{\mathrm{R}}^{a}\right)^{\mathrm{T}}($ each consisting of 2 two-component Weyl spinors $\left.\psi_{\mathrm{L}}, \psi_{\mathrm{R}}\right)$ into one collective $4 N_{\mathrm{f}}$-component spinor

$$
\Psi:=\left(\psi_{\mathrm{L}}^{1}, \psi_{\mathrm{R}}^{1}, \ldots, \psi_{\mathrm{L}}^{N_{\mathrm{f}}}, \psi_{\mathrm{R}}^{N_{\mathrm{f}}}\right)^{\mathrm{T}}
$$

(consisting of $2 N_{\mathrm{f}}$ two-component Weyl spinors), the theory is invariant under

$$
\Psi \mapsto \mathcal{U} \Psi, \quad \bar{\Psi} \mapsto \bar{\Psi} \mathcal{U}^{\dagger}, \quad \mathcal{U} \in \mathrm{U}\left(2 N_{\mathrm{f}}\right),
$$

where the entries of the unitary $\left(2 N_{\mathrm{f}}\right) \times\left(2 N_{\mathrm{f}}\right)$ matrix $\mathcal{U}$ are complex numbers times the $2 \times 2$ identity matrix $\mathbb{1}_{2}$.

Because of the reducible representation of the Dirac algebra, discrete transformations can be implemented in various ways [26]. Let us define

$$
\begin{array}{ll}
C: \psi \mapsto\left(\bar{\psi} C_{\xi}\right)^{\mathrm{T}}, \bar{\psi} \mapsto-\left(C_{\xi}^{\dagger} \psi\right)^{\mathrm{T}} & \text { (charge conjugation), } \\
\mathcal{P}: \psi \mapsto P_{\zeta} \psi, \bar{\psi} \mapsto \bar{\psi} P_{\zeta}^{\dagger} & \text { (parity inversion), } \\
\mathcal{T}: \psi \mapsto T_{\eta} \psi, \bar{\psi} \mapsto \bar{\psi} T_{\eta}^{\dagger} & \text { (time reversal), }
\end{array}
$$

where $C$ and $\mathcal{P}$ are unitary and $\mathcal{T}$ is antiunitary. The arguments of the transformed fields are $\tilde{x}:=\left(-x_{1}, x_{2}, x_{3}\right)$ in the case of parity, and $\hat{x}:=\left(x_{1}, x_{2},-x_{3}\right)$ in the case of time reversal. $C_{\xi}, P_{\zeta}$, and $T_{\eta}$ are unitary $4 \times 4$ matrices given by

$$
\begin{aligned}
C_{\xi} & =\frac{1}{2}\left[(1+\xi) \gamma_{2} \gamma_{4}+\mathrm{i}(1-\xi) \gamma_{2} \gamma_{5}\right], \\
P_{\zeta} & =\frac{1}{2}\left[(1+\zeta) \gamma_{1} \gamma_{4}+\mathrm{i}(1-\zeta) \gamma_{1} \gamma_{5}\right], \\
T_{\eta} & =\frac{1}{2}\left[(1+\eta) \gamma_{1}+\mathrm{i}(1-\eta) \gamma_{2} \gamma_{3}\right],
\end{aligned}
$$

depending on the pure phases $\xi, \zeta, \eta$ with $|\xi|=|\zeta|=|\eta|=1$. Recall that $\gamma_{1}$ and $\gamma_{3}$ are antisymmetric and purely imaginary, whereas $\gamma_{2}, \gamma_{4}$, and $\gamma_{5}$ are symmetric and real. We thus see that on the classical level our theory is invariant under any of the discrete transformations $C, \mathcal{P}$, and $\mathcal{T}$ individually, irrespective of the values of the phases $\xi, \zeta$, and $\eta$. 
Let us now consider building blocks of the effective action, starting at the two-fermion level. There are, in fact, four possible mass terms $\bar{\psi} \tau_{j} \psi$ with $\tau_{j}$ given in Eq. (9) and diagonal flavor structure. (We shall suppress the flavor index $a$ as long as it is not needed.) However, the term $\bar{\psi}\left(\mathrm{i} m+m^{\prime} \gamma_{4}\right) \psi$ transforms under $\psi \mapsto \mathrm{e}^{\mathrm{i} \alpha \gamma_{4}} \psi$ into a parity even mass term $\propto \bar{\psi} \psi$ if $\alpha$ is chosen to satisfy $2 \alpha=\arctan \left(\mathrm{m}^{\prime} / \mathrm{m}\right)$. The analogous statement holds for the mass term involving $\gamma_{5}$. More generally, any mass term can be transformed by a $\mathrm{U}\left(2 N_{\mathrm{f}}\right)$ rotation intd 2

$$
\mathrm{i} \bar{\psi}\left(m+\tilde{m} \gamma_{45}\right) \psi
$$

A dynamically generated mass $m \neq 0$ spontaneously breaks the $\mathrm{U}\left(2 N_{\mathrm{f}}\right)$ symmetry down to a residual $\mathrm{U}_{\mathbb{1}+\gamma_{45}}\left(N_{\mathrm{f}}\right) \otimes$ $\mathrm{U}_{\mathbb{1}-\gamma_{45}}\left(N_{\mathrm{f}}\right) \subsetneq \mathrm{U}\left(2 N_{\mathrm{f}}\right)$ generated by $\lambda_{b} \otimes\left(\mathbb{1} \pm \gamma_{45}\right), b=1, \ldots, N_{\mathrm{f}}^{2}$ (c.f. Eq. (10), but leaves the discrete space-time symmetries $\mathcal{C}, \mathcal{P}$, and $\mathcal{T}$ intact, in agreement with the analogous discussion in the context of $\mathrm{QED}_{3}$ [17]. A nonvanishing mass $\tilde{m}$ in contrast does not break the $\mathrm{U}\left(2 N_{\mathrm{f}}\right)$ symmetry, since $\gamma_{45}$ anticommutes with $\gamma_{4}$ and $\gamma_{5}$. However, as can be read off from Eqs. 12 14, such a mass term is odd under parity inversion since $\gamma_{45}$ anticommutes with $P_{\zeta}$. Because of $\left\{\gamma_{45}, C_{\xi}\right\}=0$ and $\left(\gamma_{45}\right)^{\mathrm{T}}=-\gamma_{45}$ it is even under charge conjugation; since $\left[\gamma_{45}, T_{\eta}\right]=0$ and $\left(\mathrm{i} \gamma_{45}\right)^{*}=\mathrm{i} \gamma_{45}$ it is also even under time reversal.

A complete basis of the $4 \times 4$ Dirac algebra is given by the 16 matrices

$$
\left\{\gamma^{A}\right\}_{A=1}^{16}=\left\{\mathbb{1}, \gamma_{\mu}, \sigma_{\mu \nu} / \sqrt{2}, \mathrm{i} \gamma_{\mu} \gamma_{4}, \mathrm{i} \gamma_{\mu} \gamma_{5}, \gamma_{4}, \gamma_{5}, \gamma_{45}\right\}
$$

where we have introduced the generators of the Lorentz transformation of the four-component Dirac spinors $\sigma_{\mu \nu}:=$ $\frac{i}{2}\left[\gamma_{\mu}, \gamma_{v}\right]$. In Eq. (16), we only count those matrices $\sigma_{\mu \nu}$ with $\mu<\nu$. A bilinear $\bar{\psi} \gamma^{A} \psi$ is invariant under $\mathrm{U}\left(2 N_{\mathrm{f}}\right)$ transformations if and only if $\gamma^{A}$ anticommutes with the generators of $\mathrm{U}_{\gamma_{4}}(1)$ and $\mathrm{U}_{\gamma_{5}}(1)$ while it commutes with the generators of $\mathrm{U}_{\mathbb{1}}(1)$ and $\mathrm{U}_{\gamma_{45}}(1)$. Obviously, this is only the case for $\bar{\psi} \gamma_{\mu} \psi$ and $\bar{\psi} \gamma_{45} \psi$. Imposing an invariance under $\mathrm{U}\left(2 N_{\mathrm{f}}\right)$ as well as $\mathcal{C}, \mathcal{P}$, and $\mathcal{T}$, there is no bilinear to zeroth derivative order. In particular, no mass term is permitted. To first order, only the standard kinetic term

$$
\mathcal{L}_{\text {kin }}=\mathrm{i} \bar{\psi} \not \partial \psi
$$

can appear. Consequently, on the level of four-fermion interactions, the Thirring interaction is not the only fermionic 4-point function in the point-like limit (i.e., with momentum independent couplings) which is invariant under the present $\mathrm{U}\left(2 N_{\mathrm{f}}\right)$ flavor symmetry and the discrete space-time symmetries. In fact, the possible interactions are

$$
S_{\mu}^{2}:=\left(\bar{\psi}^{a} \gamma_{\mu} \psi^{a}\right)^{2} \quad \text { and } \quad S^{2}:=\left(\bar{\psi}^{a} \gamma_{45} \psi^{a}\right)^{2} \text {, }
$$

\footnotetext{
2 Note that due to our chiral conventions a nonzero expectation value $\langle\bar{\psi} \psi\rangle$ or $\left\langle\bar{\psi} \gamma_{45} \psi\right\rangle$ is purely imaginary [40].
}

as well as the two interaction terms with non-singlet flavor structure

$$
\begin{gathered}
V^{2}:=\left(\bar{\psi}^{a} \psi^{b}\right)^{2}-\left(\bar{\psi}^{a} \gamma_{4} \psi^{b}\right)^{2}-\left(\bar{\psi}^{a} \gamma_{5} \psi^{b}\right)^{2}+\left(\bar{\psi}^{a} \gamma_{45} \psi^{b}\right)^{2}, \\
V_{\mu}^{2}:=\left(\bar{\psi}^{a} \gamma_{\mu} \psi^{b}\right)^{2}+\left(\bar{\psi}^{a} \frac{\sigma_{\mu v}}{\sqrt{2}} \psi^{b}\right)^{2}-\left(\bar{\psi}^{a} \mathrm{i} \gamma_{\mu} \gamma_{4} \psi^{b}\right)^{2}-\left(\bar{\psi}^{a} \mathrm{i} \gamma_{\mu} \gamma_{5} \psi^{b}\right)^{2},
\end{gathered}
$$

where we define $\left(\bar{\psi}^{a} \psi^{b}\right)^{2} \equiv \bar{\psi}^{a} \psi^{b} \bar{\psi}^{b} \psi^{a}$, etc. However, the terms in Eqs. (19), 20) are not independent of those in Eq. (18) but can be mapped onto each other by means of Fierz transformations. We find that the scalar/pseudoscalar interaction in Eq. 19] is equal to $V^{2}=-S_{\mu}^{2}-S^{2}$ and the axial/vector-type interaction in (20) obeys $V_{\mu}^{2}=S_{\mu}^{2}-3 S^{2}$; see Appendix A. This generalizes the discussion of [7] to larger flavor number $N_{\mathrm{f}}$.

To summarize: in addition to the Thirring coupling $S_{\mu}^{2}$, a second point-like linearly independent four-fermi coupling $S^{2}$ satisfies the symmetries of the Thirring model. In an RG analysis, it has to be included on the same fundamental level as the Thirring interaction.

\section{FERMIONIC RG FLOW}

All physical information of a quantum field theory is stored in correlation functions which in turn can be extracted from a generating functional. By a Legendre transform of the latter one obtains the effective action $\Gamma$, which governs the dynamics of the field expectation value, taking the effects of all quantum fluctuations into account. In other words, a given theory is solved, once $\Gamma$ is computed. Instead of integrating out all fluctuations at once, we can implement Wilson's idea of integrating out modes momentum shell by momentum shell, leading us to the scale dependent effective average action $\Gamma_{k}$, with a momentum-shell parameter $k . \quad \Gamma_{k}$ corresponds to the bare action $S=\int \mathrm{d}^{3} x \mathcal{L}$ for $k$ approaching the UV cut-off $\Lambda$, while the full quantum action $\Gamma$ is approached for $k \rightarrow 0$. The scale dependence of $\Gamma_{k}$ (as a functional of only fermionic degrees of freedom in our case) is governed by the Wetterich equation [38]

$$
\partial_{t} \Gamma_{k}[\bar{\psi}, \psi]=-\frac{1}{2} \operatorname{Tr}\left(\frac{\partial_{t} R_{k}}{\Gamma_{k}^{(2)}[\bar{\psi}, \psi]+R_{k}}\right), \quad \partial_{t} \equiv k \frac{\partial}{\partial k},
$$

where the trace is meant to be taken over all internal degrees of freedom (flavor, spinor, momentum). Here, $\Gamma_{k}^{(2)}[\bar{\psi}, \psi]$ is the second functional derivative with respect to $\bar{\psi}$ and $\psi$, and $R_{k}$ denotes a momentum-dependent regulator function, ensuring that IR modes below the momentum scale $k$ are suppressed. The minus sign on the right hand side of Eq. 21) is due to the Grassmann nature of $\bar{\psi}$ and $\psi$. For reviews, see e.g. [41, 42]. With the Wetterich equation being an exact equation, consistent approximation schemes can be devised that allow for a systematic nonperturbative investigation of the given model. In this work, we use a simple derivative expansion of the effective action in terms of purely fermionic degrees of freedom 
with point-like interactions,

$$
\begin{aligned}
\Gamma_{k}[\bar{\psi}, \psi]=\int \mathrm{d}^{3} x\left\{Z_{k} \bar{\psi}^{a} \mathrm{i} \not \partial \psi^{a}+\frac{\tilde{\bar{g}}_{k}}{2 N_{\mathrm{f}}}\left(\bar{\psi}^{a} \gamma_{45} \psi^{a}\right)^{2}\right. \\
\left.+\frac{\bar{g}_{k}}{2 N_{\mathrm{f}}}\left(\bar{\psi}^{a} \gamma_{\mu} \psi^{a}\right)^{2}\right\}
\end{aligned}
$$

In addition to the interaction terms discussed above, we have included a wave function renormalization $Z_{k}$. All parameters in the effective average action are understood to be scale dependent which is indicated by the momentum-scale index $k$. This truncation corresponds to a next-to-leading order derivative expansion, which can consistently be extended to higher orders and thus defines a systematic nonperturbative approximation scheme. As discussed in the previous section, the truncation (22) represents a full basis of fermionic 4-point functions in the point-like limit, which are compatible with the present chiral and discrete symmetries. Such a point-like truncation can be a reasonable approximation in the chirally symmetric regime, as has been quantitatively confirmed for the zero-temperature chiral phase transition in many flavor QCD [33].

Inserting Eq. (22) into Eq. (21), we obtain the flow equations (i.e., $\beta$ functions) for the 4 -fermi couplings $\bar{g}_{k}$ and $\tilde{\bar{g}}_{k}$ and the wave function renormalization $Z_{k}$ via suitable projections onto the associated operators. For the explicit computations, we refer the reader to Appendix B. In terms of renormalized fields

$$
\psi \mapsto Z_{k}^{-1 / 2} \psi_{k}, \quad \bar{\psi} \mapsto Z_{k}^{-1 / 2} \bar{\psi}_{k}
$$

and dimensionless renormalized couplings

$$
g=Z_{k}^{2} k^{-1} \bar{g}_{k}, \quad \tilde{g}=Z_{k}^{2} k^{-1} \tilde{\bar{g}}_{k},
$$

we obtain the beta functions as

$$
\begin{aligned}
& \partial_{t} \tilde{g}=\tilde{g}-\frac{4 \ell_{1}^{(\mathrm{F})}}{\pi^{2}}\left[\frac{2 N_{\mathrm{f}}-1}{2 N_{\mathrm{f}}} \tilde{g}^{2}-\frac{3}{2 N_{\mathrm{f}}} \tilde{g} g-\frac{1}{N_{\mathrm{f}}} g^{2}\right], \\
& \partial_{t} g=g+\frac{4 \ell_{1}^{(\mathrm{F})}}{\pi^{2}}\left[\frac{1}{2 N_{\mathrm{f}}} \tilde{g} g+\frac{2 N_{\mathrm{f}}+1}{6 N_{\mathrm{f}}} g^{2}\right] .
\end{aligned}
$$

Within the present truncation of point-like interactions, the anomalous dimension remains $\eta_{k}:=-\partial_{t} \ln Z_{k} \equiv 0$. These flows involve the threshold function $\ell_{1}^{(\mathrm{F})}$ which encodes the details of the regularization scheme as specified by the dimensionless regulator shape function $r\left(q^{2} / k^{2}\right)$ defined by $R_{k}(q)=$ $Z_{k} \phi r\left(q^{2} / k^{2}\right)$,

$$
\ell_{1}^{(\mathrm{F})}:=-\frac{\partial}{\partial k} \int_{0}^{\Lambda} \mathrm{d}|q| \frac{1}{\left[1+r\left(q^{2} / k^{2}\right)\right]^{2}} .
$$

For a given regulator function, this integral in the present truncation boils down to a simple number. For instance, for the sharp cut-off

$$
r^{\mathrm{sc}}\left(q^{2} / k^{2}\right)= \begin{cases}\infty & \text { for } q^{2}<k^{2} \\ 0 & \text { for } q^{2}>k^{2}\end{cases}
$$

we obtain $\ell_{1}^{(\mathrm{F})}=1$. For a linear cut-off which satisfies a regulator optimization criterion [43],

$$
r^{\mathrm{opt}}\left(q^{2} / k^{2}\right)=\left(\sqrt{\frac{k^{2}}{q^{2}}}-1\right) \Theta\left(1-q^{2} / k^{2}\right)
$$

we obtain $\ell_{1}^{(\mathrm{F})}=2 / 3$. By another rescaling $g \mapsto g \pi^{2} / 4 \ell_{1}^{(\mathrm{F})}$ and $\tilde{g} \mapsto \tilde{g} \pi^{2} / 4 \ell_{1}^{(\mathrm{F})}$, this multiplicative regulator dependence drops out,

$$
\begin{aligned}
& \partial_{t} \tilde{g}=\tilde{g}-\frac{2 N_{\mathrm{f}}-1}{2 N_{\mathrm{f}}} \tilde{g}^{2}+\frac{3}{2 N_{\mathrm{f}}} \tilde{g} g+\frac{1}{N_{\mathrm{f}}} g^{2}, \\
& \partial_{t} g=g+\frac{1}{2 N_{\mathrm{f}}} \tilde{g} g+\frac{2 N_{\mathrm{f}}+1}{6 N_{\mathrm{f}}} g^{2} .
\end{aligned}
$$

For $N_{\mathrm{f}}=1$, our result coincides with the result found via a perturbative RG approach [7] in the context of interacting electrons on the honeycomb lattice.

\section{FIXED POINTS AND CRITICAL EXPONENTS}

From the coupling flows, it is straightforward to analyze the fixed-point structure in order to study possible asymptotically safe UV trajectories of the RG flow. A fixed point $g^{*}$ is defined by

$$
\forall i: \quad \beta_{i}\left(g_{1}^{*}, g_{2}^{*}, \ldots\right)=0
$$

with $\beta_{i} \equiv \partial_{t} g_{i}$. Whereas the fixed-point values are regulatorscheme dependent, see above, the linearized flow in the vicinity of the fixed point is universal as quantified by the critical exponents. More specifically, the Jacobian $B_{i}{ }^{j}$ of the linearized flow near a fixed point,

$$
\partial_{t} g_{i}=B_{i}{ }^{j}\left(g_{j}-g_{j}^{*}\right)+\ldots, \quad B_{i}^{j}=\left.\frac{\partial \beta_{i}}{\partial g_{j}}\right|_{g=g^{*}},
$$

defines the stability matrix. The associated eigenvectors $v$ govern the evolution of small deviations from the fixed point according to $\partial_{t} v=B v=-\Theta v$. The corresponding eigenvalues (including a minus sign) $\Theta$ are universal and can be associated with thermodynamic critical exponents if the fixed point corresponds to a critical point of a 2 nd order phase transition. For brevity, all $\Theta$ 's are referred to as critical exponents. The solution to the linearized flow $v \propto k^{-\Theta}$ implies that positive $\Theta>0$ correspond to RG relevant, i.e., infrared repulsive, directions and negative $\Theta<0$ correspond to RG irrelevant, i.e., infrared attractive, directions.

For the present set of flow equations, a general property of the fixed points and their critical exponents can be proven [44]: the beta functions all are of the form

$$
\beta_{i}=g_{i}+g_{k} A_{i}^{k l} g_{l} \quad \Rightarrow \quad B_{i}{ }^{j}=\delta_{i}{ }^{j}+2 g_{k}^{*} A_{i}^{k j},
$$

with matrices $A_{i}^{k l}$ which are symmetric in the upper indices. We now see that for every interacting fixed point $g^{*} \neq 0$ the 


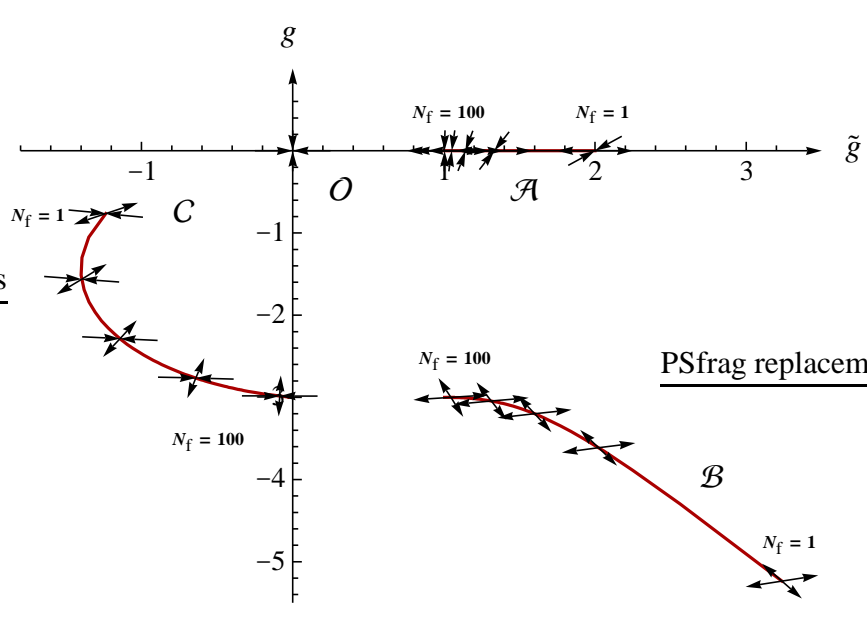

FIG. 1. Positions of RG fixed points and (ir-)relevant directions for flavor numbers $N_{\mathrm{f}}=1,2,4,10,100$. Arrows denote the RG flow towards the IR. In agreement with Eq. 35, one relevant direction of every interacting fixed point points towards the Gaußian fixed point $O$.

fixed-point vector itself is an eigenvector of $B, v=g^{*}$, with the critical exponent $\Theta=1$,

$$
B_{i}{ }^{j} g_{j}^{*}=g_{i}^{*}+2 g_{k}^{*} A_{i}^{k j} g_{j}^{*}=-g_{i}^{*}
$$

Here, we have made use of the fixed point equation $\beta_{j}\left(g^{*}\right)=0$. We conclude that every fixed point besides the Gaußian fixed point $g^{*}=0$ has at least one relevant and thus infrared repulsive direction. Each non-Gaußian fixed point is therefore a candidate for a possible UV completion, potentially defining an own universality class [44]. For the Gaußian fixed point $g^{*}=0$, the stability matrix is just the identity $B_{i}{ }^{j}=\delta_{i}{ }^{j}$, such that the Gaußian fixed point is infrared attractive in every direction with $\Theta=-1$, giving rise to only trivial theories at long ranges.

For fixed $g_{j \neq i}$, the beta function $\beta_{i}$ corresponds graphically to a parabola, such that we expect for our truncation exactly $2^{2}=4$ (possibly complex or degenerate) solutions of the fixed-point equations. For any $N_{\mathrm{f}} \in \mathbb{N}$, we find them to be real and non-degenerate; the explicit solutions $\left(\tilde{g}^{*}, g^{*}\right)$ for the Gaußian fixed point $O$ and the three non-Gaußian fixed points $\mathcal{A}, \mathcal{B}, \mathcal{C}$ are

$$
\begin{aligned}
\mathcal{O}: & (0,0) \\
\mathcal{A}: & \left(\frac{2 N_{\mathrm{f}}}{2 N_{\mathrm{f}}-1}, 0\right) \\
\mathcal{B}: & \left(\frac{N_{\mathrm{f}}\left(14-7 N_{\mathrm{f}}+2 N_{\mathrm{f}}^{2}+\left(1+2 N_{\mathrm{f}}\right) \sqrt{16+28 N_{\mathrm{f}}+N_{\mathrm{f}}^{2}}\right)}{-5+8 N_{\mathrm{f}}+2 N_{\mathrm{f}}^{2}+4 N_{\mathrm{f}}^{3}}\right. \\
& \left.-\frac{12 N_{\mathrm{f}}^{2}}{4+4 N_{\mathrm{f}}+4 N_{\mathrm{f}}^{2}-\sqrt{16+28 N_{\mathrm{f}}+N_{\mathrm{f}}^{2}}}\right)
\end{aligned}
$$

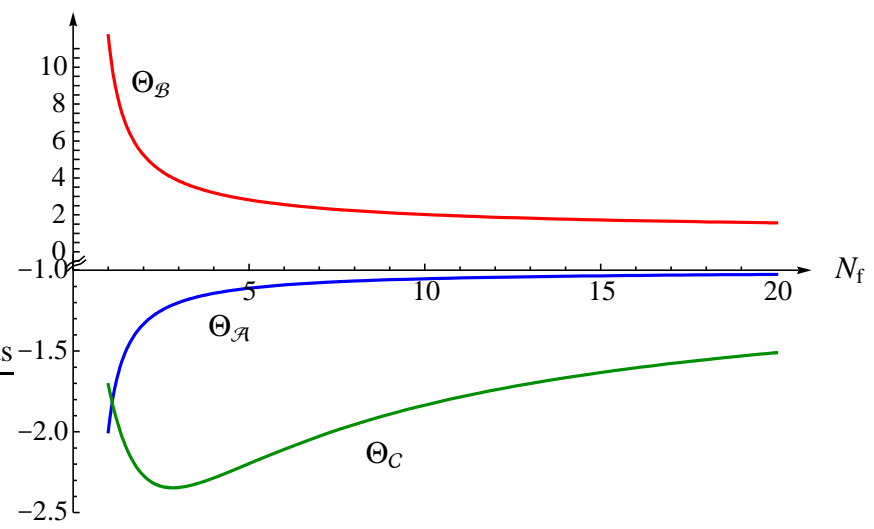

FIG. 2. Critical exponents $\Theta$ as a function of flavor number $N_{\mathrm{f}}$ for the interacting fixed points $\mathcal{A}, \mathcal{B}, C$. The value of the corresponding other critical exponent is $\Theta=1$ for all $N_{\mathrm{f}}$. Note that the scale of the vertical axis changes at $\Theta=-1$.

$$
\begin{aligned}
C: & \left(\frac{N_{\mathrm{f}}\left(14-7 N_{\mathrm{f}}+2 N_{\mathrm{f}}^{2}-\left(1+2 N_{\mathrm{f}}\right) \sqrt{16+28 N_{\mathrm{f}}+N_{\mathrm{f}}^{2}}\right)}{-5+8 N_{\mathrm{f}}+2 N_{\mathrm{f}}^{2}+4 N_{\mathrm{f}}^{3}}\right. \\
& \left.-\frac{12 N_{\mathrm{f}}^{2}}{4+4 N_{\mathrm{f}}+4 N_{\mathrm{f}}^{2}+\sqrt{16+28 N_{\mathrm{f}}+N_{\mathrm{f}}^{2}}}\right)
\end{aligned}
$$

It is straightforward to derive the eigenvectors and eigenvalues of the stability matrix (and thus the critical exponents and the RG (ir-)relevant directions) analytically; but as the general formulas may not provide much physical insight, we present the results graphically: in Fig. 1, we plot the positions of the fixed points in theory space (spanned by the two couplings $\tilde{g}$ and $g$ ) together with the corresponding eigenvectors of the stability matrix $B_{i}{ }^{j}$ for various flavor numbers $N_{\mathrm{f}}$. In Fig. 2, the (non-trivial) critical exponents $\Theta$ for the interacting fixed points are given as a function of $N_{\mathrm{f}}$. For any $N_{\mathrm{f}}$, fixed point $\mathcal{B}$ has two RG relevant directions, whereas the interacting fixed points $\mathcal{A}$ and $C$ have one relevant and one irrelevant direction. As the number of relevant directions corresponds to the number of physical parameters to be fixed, theories emanating from $\mathcal{A}$ and $C$ are fully determined, once the initial condition for this relevant direction is fixed. In the sense of dimensional transmutation, fixing this one parameter can be viewed as fixing a total scale for the system. Therefore, theories belonging to these universality classes defined by $\mathcal{A}$ and $C$ are fully predictive, once a global scale is fixed. Theories emanating from $\mathcal{B}$ are fixed by a mass scale and one further parameter, e.g., a dimensionless coupling ratio, whereas $O$ does not support an interacting system at long ranges.

At this point, let us already stress that no interacting fixed point is on the pure Thirring axis $(\tilde{g}=0)$ for any finite $N_{\mathrm{f}}$. In fact, as discussed below, we associate the Thirring universality class with fixed point $C$ which approaches the pure Thirring coupling only in the asymptotic limit $N_{\mathrm{f}} \rightarrow \infty$. For any finite $N_{\mathrm{f}}$, the renormalized UV trajectory of the Thirring model will have to pass through the full two-dimensional coupling plane, even though the long-range physics does depend only on one physical parameter (e.g., the value of the Thirring coupling at a certain scale). 


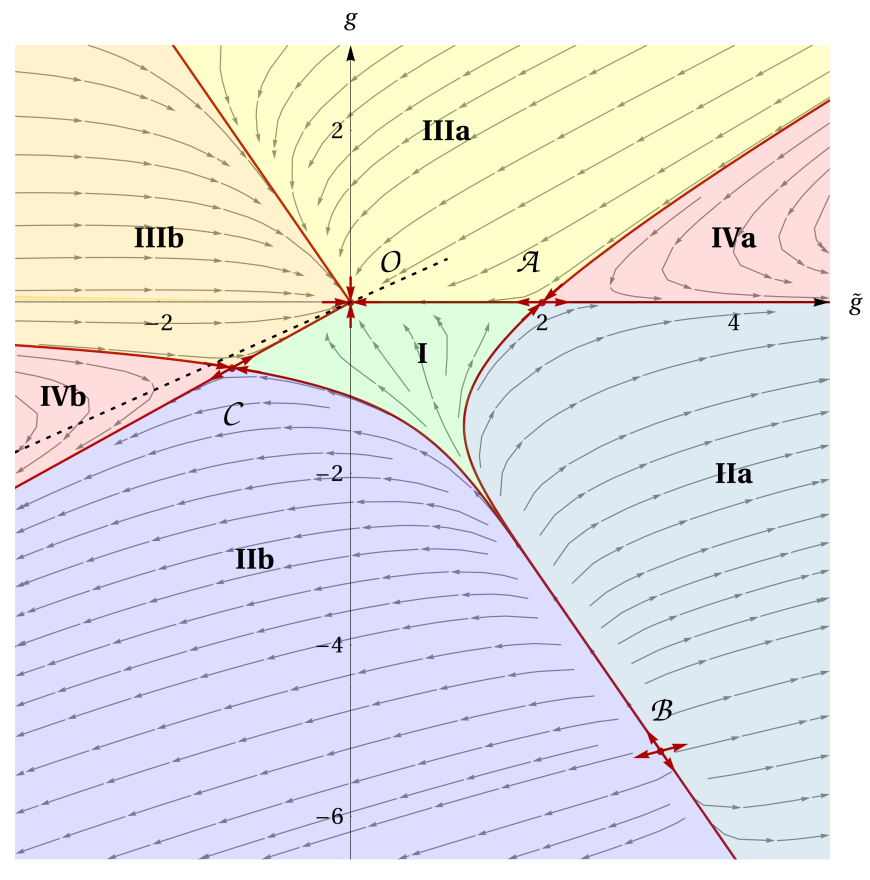

FIG. 3. Classification of Thirring-like 4-fermi theories determined by the fixed-point positions and the corresponding RG trajectories (arrows denote the flow towards the IR). Red (solid) lines depict separatrices that interpolate between fixed points and separate different regions. The vertical axis $\tilde{g}=0$ corresponds to models with a pure Thirring coupling. The dashed line $(g=\tilde{g} / 2)$ marks a generalized NJL model, see text.

\section{PHASE TRANSITIONS AND LONG-RANGE PHYSICS}

A technical means for the discussion of long-range phases are the separatrices, i.e., those RG trajectories that interpolate between two fixed points. They subdivide the theory space into separate flow regions, providing a classification which can potentially be related to spontaneous symmetry breaking in the long-range limit. At this point, we stress that the fermionic truncation is not sufficient for a complete discussion of long-range physics which is expected to be dominated by composite bosonic degrees of freedom such as condensates and excitations on top of condensates.

Let us start with a closer look at the one-flavor case $N_{\mathrm{f}}=1$. The separatrices subdivide the theory space into distinct sections defined by their IR and UV behavior; in Fig. 3, we plot the RG flow using Eqs. 30-31. A classification of RG trajectories is listed in Tab. II. The IR behavior of the theories in the regions I, IIIa and IIIb is governed by the Gaußian fixed point $O$. In these regions, both couplings $\tilde{g}$ and $g$ are irrelevant, leading to non-interacting theories in the IR. The regions IIa and IVa are characterized by an irrelevant Thirring coupling $g, \lim _{k \rightarrow 0} g=0$. We thus expect that the bosonic channel $S \sim \bar{\psi}^{a} \gamma_{45} \psi^{a}$ becomes critical at a sufficiently large $\tilde{g}$, dynamically generating a parity breaking mass $\tilde{m} \propto\left\langle\bar{\psi} \gamma_{45} \psi\right\rangle$. If so, the fixed point $\mathcal{A}$ governs the spontaneous breaking of parity, potentially being associated with a 2 nd order phase transition defining a new universality class.
TABLE I. Classification of all RG trajectories, c.f. Fig. 3

\begin{tabular}{cccc}
\hline \hline Type & $\begin{array}{c}\text { UV behavior } \\
\lim _{k \rightarrow 0}(\tilde{g}, g)\end{array}$ & $\begin{array}{c}\text { IR behavior } \\
\lim _{k \rightarrow \Lambda}(\tilde{g}, g)\end{array}$ & universality class \\
\hline I & $\mathcal{B}$ & $O$ & non interacting \\
I-IIa separ. & $\mathcal{B}$ & $\mathcal{A}$ & \\
I-IIb separ. & $\mathcal{B}$ & $\mathcal{C}$ & \\
I-IIIa separ. & $\mathcal{A}$ & $O$ & non interacting \\
I-IIIb separ. & $\mathcal{C}$ & $O$ & non interacting \\
IIa & $\mathcal{B}$ & $(\infty, 0)$ & parity breaking \\
IIa-IIb separ. & $\mathcal{B}$ & $\mathcal{B} \cdot \infty$ & \\
IIa-IVa separ. & $\mathcal{A}$ & $(\infty, 0)$ & parity breaking \\
IIb & $\mathcal{B}$ & $\mathcal{C} \cdot \infty$ & chirality breaking \\
IIb-IVb separ. & $\mathcal{C}$ & $\mathcal{C} \cdot \infty$ & chirality breaking \\
IIIa & $(-C) \cdot \infty$ & $O$ & non interacting \\
IIIa-IIIb separ. & $(-\mathcal{B}) \cdot \infty$ & $O$ & non interacting \\
IIIa-IVa separ. & $(-\mathcal{C}) \cdot \infty$ & $\mathcal{A}$ & \\
IIIb & $(-\infty, 0)$ & $O$ & non interacting \\
IIIb-IVb separ. & $(-\infty, 0)$ & $\mathcal{C}$ & \\
IVa & $(-\mathcal{C}) \cdot \infty$ & $(\infty, 0)$ & parity breaking \\
IVb & $(-\infty, 0)$ & $C \cdot \infty$ & chirality breaking \\
\hline \hline
\end{tabular}

By contrast, both $\tilde{g}$ and $g$ diverge in the regions IIb and $\mathrm{IVb}$ in the infrared limit. To interpret this behavior, let us (for arbitrary $N_{\mathrm{f}} \geq 1$ ) rewrite the interaction terms by means of the Fierz theorem as

$$
\begin{aligned}
& \frac{\tilde{g}}{2 N_{\mathrm{f}}} S^{2}+\frac{g}{2 N_{\mathrm{f}}} S_{\mu}^{2}= \frac{1}{4 N_{\mathrm{f}}}\left\{(2 g-\tilde{g})\left(\bar{\psi}^{a} \gamma_{\mu} \psi^{a}\right)^{2}\right. \\
&-\tilde{g}\left[\left(\bar{\psi}^{a} \psi^{a}\right)^{2}-\left(\bar{\psi}^{a} \gamma_{4} \psi^{a}\right)^{2}-\left(\bar{\psi}^{a} \gamma_{5} \psi^{a}\right)^{2}\right] \\
&\left.+\tilde{g}\left[\left(\bar{\psi}^{a} \gamma_{45} \psi^{a}\right)^{2}-\left(\bar{\psi}^{a} \gamma_{45} \psi^{b}\right)^{2}\right]\right\},
\end{aligned}
$$

cf. Eqs. (18) 20 and App. A. In the one-flavor case $N_{\mathrm{f}}=1$, the last term (proportional to $+\tilde{g}$ ) vanishes. The interaction then simply is a linear combination of the Thirring interaction with coupling $2 g-\tilde{g}$ and a generalized Nambu-JonaLasinio (NJL)-type [45] interaction with coupling $-\tilde{g}$. Along the straight line through $O$ and $C$ which may govern the IR behavior of the trajectories IIb and IVb the NJL-type interaction in fact dominates, $|\tilde{g}| /|2 g-\tilde{g}| \approx 4.24 \gg 1$. To illustrate this, we have also plotted in Fig. 3 the line of vanishing Thirring interaction $2 g-\tilde{g}=0$ in the Fierz-transformed form as a dashed line. This resulting NJL-type line is fairly close to the separatrix through $O$ and $C$ (red line). We thus expect that at a large coupling on this separatrix the NJL-type channel eventually becomes critical, inducing a symmetry-broken state with

$$
m \propto\langle\bar{\psi} \psi\rangle \neq 0 .
$$

This is equivalent to saying that $\left\langle\bar{\psi} \gamma_{4} \psi\right\rangle \neq 0$ or $\left\langle\bar{\psi} \gamma_{5} \psi\right\rangle \neq 0$ is expected to be favored in this state. For $N_{\mathrm{f}}=1$, we therefore identify the fixed point $C$ with the critical point governing the phase transition into the chiral symmetry broken phase for all theories of the regions IIb and IVb, in agreement with [7]. This is precisely the behavior which is expected in 


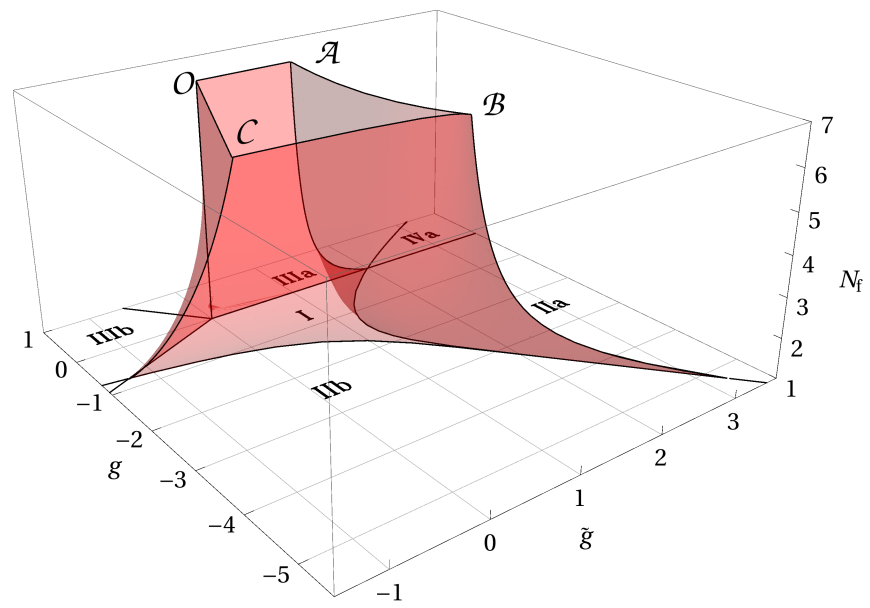

FIG. 4. Fixed-point positions and separatrices in the $(g, \tilde{g})$ coupling plane as a function of flavor number for $1 \leq N_{\mathrm{f}} \leq 7$. The horizontal slice at $N_{\mathrm{f}}=1$ is equivalent to Fig. 3

the Thirring model, hence we associate all trajectories emanating from $C$ with UV complete fully renormalized versions of the $3 d$ Thirring model.

Provided the last term in Eq. (40) proportional to $+\tilde{g}$ does not dominate even for higher number of flavors, we may extend the preceding discussion to larger $N_{\mathrm{f}} \geq 1$. In Fig. 4, we show how the positions of the fixed points and the separatrices behave for increasing flavor number. For $N_{\mathrm{f}} \rightarrow \infty$ the region I turns into a rectangle with the vertices $(\tilde{g}, g)=(0,0)$ and $(1,-3)$. The Thirring fixed point $C$ hence moves to $(0,-3)$ and becomes a pure Thirring coupling, c.f. also Fig. 11 Thus, the IR attractive line $O C$ approaches the Thirring axis $\tilde{g}=0$ for larger flavor number.

Along this line of coupling values, we expect the flow to be dominated by the vector channel $\bar{\psi}^{a} \gamma_{\mu} \psi^{a}$ which agrees precisely with the dominant bosonic degree of freedom in a large$N_{\mathrm{f}}$ analysis [24-27]. As there is no chiral symmetry breaking at large $N_{\mathrm{f}}$, it is natural to expect a quantum phase transition to occur for increasing $N_{\mathrm{f}}$ while the line $O C$ undergoes a transition from the NJL-regime $g \sim \tilde{g} / 2$ to the large- $N_{\mathrm{f}}$ regime where $\tilde{g}=0$. Unfortunately, a more quantitative picture of this quantum phase transition is difficult to obtain in the purely fermionic language. A quantitative RG analysis requires the inclusion of dynamical chiral (i.e., NJL-type) and vector bosonic degrees of freedom in order to study the interplay of these competing orders as a function of $N_{\mathrm{f}}$. This is left for future work.

For the remainder of this section, we shall be satisfied with a simple estimate of the transition region. As a rough criterion, let us determine the flavor number where the separatrix $O C$ is half way in-between the generalized NJL model on the one hand and the pure Thirring coupling on the other hand. This value of $N_{\mathrm{f}}$ follows from

$$
\left.\frac{|\tilde{g}|}{|2 g-\tilde{g}|}\right|_{C}=\frac{3}{N_{\mathrm{f}}-7+\sqrt{16+28 N_{\mathrm{f}}+N_{\mathrm{f}}^{2}}} \approx 1,
$$

and is given by $N_{\mathrm{f}} \approx 7 / 4$. We stress that this number should not be viewed as a direct estimate of $N_{\mathrm{f}}^{\mathrm{cr}}$, as the onset of true

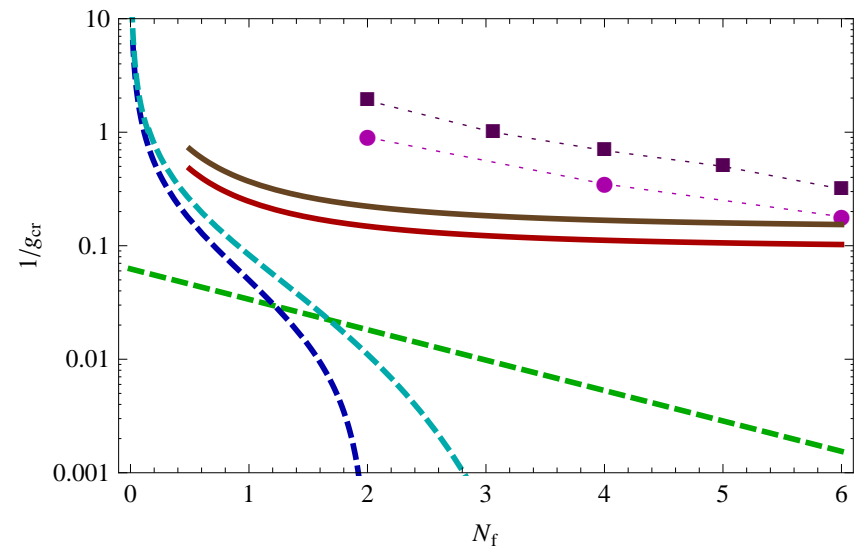

FIG. 5. Comparison of non-universal critical Thirring couplings from different methods. Solid lines display the critical couplings from this work taken as the $g$ coordinate of the intersection point of the separatrix $\mathcal{B C}$ with the Thirring axis $\tilde{g}=0$ for the sharp cutoff (upper solid/brown line) or the linear regulator (lower solid/red line). Dotted: Monte Carlo results (magenta with circles: [30], purple with squares: [31]). Dashed: DSE approaches (from right to left: green [27], cyan [28] and blue [29]).

critical behavior can easily provide further correction factors of order $O(1)$.

It is instructive to compare our results with those obtained by other methods such as Monte Carlo simulations or truncated DSEs. A variety of studies have computed the modulus of the critical Thirring coupling which is a necessarily required for chiral symmetry breaking (but not sufficient beyond the critical flavor number). Two cautionary remarks are in order: first of all, these critical couplings similarly as the fixed-point values are not universal, such that the choice of the regularization scheme can have a strong quantitative influence on the estimated values. Second, most other studies have defined the microscopic Thirring model exactly on the Thirring axis $\tilde{g}=0$; in principle, the full coupling plane has to be considered such that the critical coupling on the axis may be different (larger in modulus) from a corresponding estimate directly at the fixed point.

To circumvent the second caveat, we also consider the coupling on the Thirring axis $\tilde{g}=0$ with an initial value of the coupling $g$ above the separatrix which interpolates between the fixed points $\mathcal{B}$ and $\mathcal{C}$. Then the theory is in the region I or IIIa, depending on the sign of $g$, such that the RG flow drives the couplings to the free theory at Gaußian fixed point $O$. Therefore, the absolute value of the $g$ coordinate of the intersection point of this separatrix with the Thirring axis provides a lower bound for the absolute value of the critical coupling $g_{\text {cr }}$ at which the $\chi$ SB phase transition occurs. We compare this lower bound for different regulator functions $R_{k}$ with the values of the critical coupling obtained by Monte Carlo simulations [30, 31] and different sequences of truncated DSE approaches [27-29]. In Fig. 5] we plot this inverse coupling $1 / g_{\text {cr }}$ for varying number of flavors $0<N_{\mathrm{f}} \leq 6$. For the sharp cut-off as well as for the linear cut-off our results lie well above the values obtained by DSE approaches but below 
the values from lattice-regularized Monte Carlo simulations. Note that similar to the lattice and one of the DSE results, we do not observe a sharp decay of the inverse critical coupling. This behavior would indicate a sharp growth of $g_{\text {cr }}$ above a certain number of flavors, corresponding to a critical flavor number in the infinite coupling limit. Instead, we observe a rather smooth dependence on $N_{\mathrm{f}}$ as on the lattice for $N_{\mathrm{f}} \lesssim 6$, which is compatible with our expectation that the quantum phase transition towards the chirally symmetric phase occurs because of competing large- $N_{\mathrm{f}}$ degrees of freedom and not because of a change in the UV critical structure.

\section{CONCLUSIONS}

In this work, we have investigated $3 d$ relativistic fermionic models in a theory space, defined by chiral and a set of discrete symmetries and point-like interactions. Even though the construction of these models has been inspired by the uniquely fixed $2 d$ Thirring model, the corresponding $3 d$ symmetries involving a reducible 4-component Dirac spinor representation enlarge the minimal coupling space and give room for a larger fixed-point structure and thus for different microscopic realizations of such fermion models.

We have classified all point-like interactions satisfying the symmetry constraints and determined their RG flow in a systematic next-to-leading order derivative expansion. The fact that leading-order and next-to-leading order results are identical as the anomalous dimension remains zero can be interpreted as a signature for the convergence of the expansion, as long as no further composite channels develop a strong RG flow.

The resulting flow equations for the two independent fermionic couplings generate a fixed-point structure of one trivial Gaußian and three interacting fixed points $\mathcal{A}, \mathcal{B}, C$ which can be classified by their critical exponents. We associate all $\mathrm{RG}$ trajectories emanating from the fixed point $C$ with fully renormalized UV complete versions of the $3 d$ Thirring model; as this fixed point has one RG relevant direction, fixing one physical scale suffices to obtain a fully IR predictive quantum field theory in the Thirring universality class.

Fixed point $\mathcal{A}$ which, incidentally, lives in an RG invariant sub-manifold of theory space (defined by $g=0$ ) is also characterized by only one RG relevant direction. From the nature of the scalar channel associated with this coupling direction, we conjecture that this fixed point can be related to a critical point of a 2 nd order phase transition to a phase with broken parity. The third interacting fixed point $\mathcal{B}$ has two relevant directions; depending on the initial conditions, theories emanating from this fixed point can flow to the Thirring phase as well as to the parity-broken or symmetric phases.

Unfortunately, our purely fermionic RG analysis does so far not permit us to reliably run towards or into the symmetrybroken phases. Such a quantitative description is required in order to analyze the true IR behavior of the Thirring phase. From the structure of the fermionic flow, in particular, from the behavior of the separatrix $O C$, we conjecture that the longrange dynamics is characterized by a competition between
NJL-type chiral condensation channels on the one hand and large- $N_{\mathrm{f}}$-type vector bosons on the other hand. As the vectorboson fluctuations generically inhibit chiral symmetry breaking, we expect the occurrence of a quantum phase transition of the Thirring model at a critical flavor number $N_{\mathrm{f}}^{\mathrm{cr}}$, separating a broken phase for small $N_{\mathrm{f}}$ from a disordered phase for large $N_{\mathrm{f}}$. Our very rough estimate of the transition region in any case is compatible with the findings from lattice simulations indicating that $N_{\mathrm{f}}^{\mathrm{cr}} \simeq 6.6[31]$.

Our results on the position of the Thirring fixed point $C$ being actually away from the pure Thirring axis $\tilde{g}=0$ for any finite $N_{\mathrm{f}}$ provokes an important comment: both lattice simulations as well as DSE studies build on a microscopic definition of the $3 d$ Thirring model which is fixed only with the Thirring coupling, i.e., by a pure bare Thirring-like action. Our fixed-point results indeed provide a fundamental justification for this, as the Thirring fixed point indeed is characterized by only one relevant direction. As long as the microscopic actions chosen in other formulations are in a sufficiently attractive domain of the Thirring fixed point, universality guarantees that the long-range physics is indeed purely governed by the Thirring fixed point. We expect this to hold also for the determination of the critical flavor number (there is no universality for quantities such as the scheme-dependent critical coupling, see Fig. 57. Nevertheless, one caveat should be emphasized: this conclusion about universality only holds, as long as the microscopic bare actions indeed are in the attractive domain of the Thirring fixed point. For instance, if by accident a lattice formulation turned out to be influenced by fixed point $\mathcal{B}$, the simulation would simply describe a model different from the Thirring model potentially exhibiting a different quantum phase transition as a function of $N_{\mathrm{f}}^{\mathrm{cr}}$. As the fixed-point positions are not universal, our results are unfortunately not directly transferable to the lattice theory space. However, provided that the fixed-point structure is qualitatively similar, our results can be taken as a support for the implicit assumption that the lattice simulations have indeed been performed in the real Thirring universality class.

Given the importance of the quantitative value of the critical flavor number $N_{\mathrm{f}}^{\mathrm{cr}}$ of the Thirring model in the light of condensed-matter applications, a natural next step of our studies will be the inclusion of composite degrees of freedom in order to study the competition among the various bosonic channels. Within the functional RG, this can conveniently be investigated by means of partial or dynamical bosonization [46]. As the problem of competing order is a paradigmatic one in (quantum) critical phenomena and statistical physics [47], we consider the relativistic $3 d$ Thirring model as an ideal test case.

\section{ACKNOWLEDGMENTS}

Helpful discussions with J. Braun, S. Hands, S. Rechenberger, D. Scherer, and M. Scherer are gratefully acknowledged. This work has been supported by the DFG under GRK 1523, FOR 723 and Gi 328/5-1. 


\section{Appendix A: Fierz transformations}

Let $\left(\gamma^{A}, \gamma^{B}\right):=\operatorname{Tr}\left(\gamma^{A} \gamma^{B}\right)$ define an inner product on the space of $4 \times 4$ Dirac matrices. The 16 matrices $\left\{\gamma^{A}\right\}$ in Eq. 16 are orthogonal with respect to this product,

$$
\operatorname{Tr}\left(\gamma^{A} \gamma^{B}\right)=4 \delta^{A B}
$$

and thus define a complete basis of the $4 \times 4$ Dirac matrices (which generically have 16 independent matrix elements),

$$
\sum_{A=1}^{16} \frac{1}{4} \gamma_{m \ell}^{A} \gamma_{i k}^{A}=\delta_{m k} \delta_{i \ell}
$$

The Fierz transformations are straightforwardly obtained by multiplying the completeness relation (A2) by each of the 4fermi terms $\bar{\psi}_{m}^{a}\left(\gamma_{A} \psi^{b}\right)_{k} \bar{\psi}_{i}^{c}\left(\gamma_{A} \psi^{d}\right)_{\ell}$ and, where appropriate, decomposing multiple products of Dirac matrices into basis elements $\gamma_{A}$. We find

$$
\left(\bar{\psi}^{a} \gamma_{A} \psi^{b}\right)\left(\bar{\psi}^{c} \gamma_{A} \psi^{d}\right)=\sum_{B=1}^{16} C_{A B}\left(\bar{\psi}^{a} \gamma_{B} \psi^{d}\right)\left(\bar{\psi}^{c} \gamma_{B} \psi^{b}\right)
$$

with

$$
\left(C_{A B}\right)=\frac{1}{4}\left(\begin{array}{cccccccc}
-1 & -1 & -1 & -1 & -1 & -1 & -1 & -1 \\
-3 & 1 & 3 & 1 & -1 & -1 & -3 & 3 \\
-1 & 1 & -1 & -1 & 1 & -1 & 1 & 1 \\
-3 & 1 & -3 & 1 & 1 & 1 & -3 & -3 \\
-3 & -1 & 3 & 1 & 1 & -1 & 3 & -3 \\
-3 & -1 & -3 & 1 & -1 & 1 & 3 & 3 \\
-1 & -1 & 1 & -1 & 1 & 1 & -1 & 1 \\
-1 & 1 & 1 & -1 & -1 & 1 & 1 & -1
\end{array}\right)
$$

and $\left(\gamma_{A}\right)=\left(\mathbb{1}, \gamma_{\mu}, \gamma_{4}, \frac{\sigma_{\mu v}}{\sqrt{2}}, \mathrm{i} \gamma_{\mu} \gamma_{4}, \mathrm{i} \gamma_{\mu} \gamma_{5}, \gamma_{45}, \gamma_{5}\right)^{\mathrm{T}}$. With these preliminaries one can simply read off the Fierz relations be- tween the invariant 4-fermi interactions in Eqs. (18), (19) and (20).

\section{Appendix B: Derivation of flow equations}

Consider the right-hand side of the flow equation (21). By introducing the derivative $\tilde{\partial}_{t}$, acting per definition only on the $t$ dependence of the regulator $R_{k}$, we may expand

$$
\begin{aligned}
\frac{\partial_{t} R_{k}}{\Gamma_{k}^{(2)}+R_{k}}= & \tilde{\partial}_{t} \ln \left(\Gamma_{k, 0}^{(2)}+R_{k}+\Delta \Gamma_{k}^{(2)}\right) \\
= & \tilde{\partial}_{t} \ln \left(\Gamma_{k, 0}^{(2)}+R_{k}\right)+\tilde{\partial}_{t}\left(\frac{\Delta \Gamma_{k}^{(2)}}{\Gamma_{k, 0}^{(2)}+R_{k}}\right) \\
& -\frac{1}{2} \tilde{\partial}_{t}\left(\frac{\Delta \Gamma_{k}^{(2)}}{\Gamma_{k, 0}^{(2)}+R_{k}}\right)^{2}+\ldots
\end{aligned}
$$

with $\Delta \Gamma_{k}^{(2)}$ containing the field-dependent parts of $\Gamma_{k}^{(2)}$, and $\Delta \Gamma_{k, 0}^{(2)}$ containing the field-independent (propagator) part, such that $\Gamma_{k}^{(2)}=\Gamma_{k, 0}^{(2)}+\Delta \Gamma_{k}^{(2)}$. The Hessian of the average effective action in terms of Fourier transformed fields $\psi(q) \equiv \psi_{q}$ and $\bar{\psi}(q) \equiv \bar{\psi}_{q}$ is given by

$$
\Gamma_{k}^{(2)}(p, q)=\left(\begin{array}{ll}
\frac{\vec{\delta}}{\delta \psi_{-p}^{a} \mathrm{~T}} \Gamma_{k} \frac{\overleftarrow{\delta}}{\delta \psi_{q}^{b}} & \frac{\vec{\delta}}{\delta \psi_{-p}^{a} \mathrm{~T}} \Gamma_{k} \frac{\overleftarrow{\delta}}{\delta \bar{\psi}_{-q}^{\mathrm{T}} \mathrm{T}} \\
\frac{\vec{\delta}}{\delta \bar{\psi}_{p}^{a}} \Gamma_{k} \frac{\overleftarrow{\delta}}{\delta \psi_{q}^{b}} & \frac{\vec{\delta}}{\delta \bar{\psi}_{p}^{a}} \Gamma_{k} \frac{\overleftarrow{\delta}}{\delta \bar{\psi}_{-q}^{b} \mathrm{~T}}
\end{array}\right)
$$

The corresponding fluctuation matrix results in

$$
\begin{aligned}
\Delta \Gamma_{k}^{(2)}(p, q)= & \frac{\tilde{\bar{g}}_{k}}{N_{\mathrm{f}}}\left(\begin{array}{cc}
-\int_{p_{1}}\left(\bar{\psi}_{p_{1}}^{a} \gamma_{45}\right)^{\mathrm{T}}\left(\bar{\psi}_{q-p-p_{1}}^{b} \gamma_{45}\right) & \int_{p_{1}}\left\{\left(\bar{\psi}_{p_{1}}^{a} \gamma_{45}\right)^{\mathrm{T}}\left(\gamma_{45} \psi_{p-q+p_{1}}^{b}\right)^{\mathrm{T}}-\left(\bar{\psi}_{p_{1}} \gamma_{45} \psi_{p-q+p_{1}}\right) \gamma_{45}^{\mathrm{T}} \delta^{a b}\right\} \\
\int_{p_{1}}\left\{\left(\bar{\psi}_{p_{1}} \gamma_{45} \psi_{p-q+p_{1}}\right) \gamma_{45} \delta^{a b}+\left(\gamma_{45} \psi_{p_{1}}^{a}\right)\left(\bar{\psi}_{q-p+p_{1}}^{b} \gamma_{45}\right)\right\} & -\int_{p_{1}}\left(\gamma_{45} \psi_{p_{1}}^{a}\right)\left(\gamma_{45} \psi_{p-q-p_{1}}^{b}\right)^{\mathrm{T}}
\end{array}\right) \\
& + \text { term with }\left(\tilde{\bar{g}}_{k}, \gamma_{45}\right) \leftrightarrow\left(\bar{g}_{k}, \gamma_{\mu}\right)
\end{aligned}
$$

with $\int_{p_{1}} \equiv \int \frac{\mathrm{d}^{3} p_{1}}{(2 \pi)^{3}}$, and for the propagator

$$
\left(\Gamma_{k, 0}^{(2)}+R_{k}\right)^{-1}=-\frac{(2 \pi)^{3} \delta_{p-q}^{(3)} \delta^{a b}}{Z_{k} q^{2}\left(1+r\left(q^{2} / k^{2}\right)\right)}\left(\begin{array}{cc}
0 & q \\
q^{\mathrm{T}} & 0
\end{array}\right)
$$

with $R_{k}(q)=Z_{k} q r\left(q^{2} / k^{2}\right)$.

Anomalous dimension. The flow of the wave function renormalization $Z_{k}$ is obtained by a suitable projection of the
Wetterich equation 21,

$$
\partial_{t} Z_{k}=\frac{1}{24} \operatorname{Tr}\left\{\left.\gamma_{\mu} \frac{\partial}{\partial p_{\mu}^{\prime}} \int_{q^{\prime}} \frac{\vec{\delta}}{\delta \bar{\psi}_{p^{\prime}}^{1}}\left[\operatorname{Tr}\left(\frac{\partial_{t} R_{k}}{\Gamma_{k}^{(2)}+R_{k}}\right)\right] \frac{\overleftarrow{\delta}}{\delta \psi_{q^{\prime}}^{1}}\right|_{\psi=\bar{\psi}=0}\right\}
$$

Using the expansion (B2), we observe that only the term linear in $\Delta \Gamma_{k}^{(2)}$ survives the projection. Since this term is traceless, the flow of the wave function renormalization in this order of 
the expansion vanishes,

$$
\eta_{k}=-\partial_{t} \ln Z_{k} \equiv 0
$$

This line of argument is reminiscent to a similar observation of the vanishing of the anomalous dimension in scalar $O(N)$ models in the symmetric regime at next-to-leading or- der derivative expansion.

4-fermi couplings. Obviously only the term in Eq. (B2) being quadratic in $\Delta \Gamma_{k}^{(2)}$ leads to 4-fermi terms on the right-hand side of the Wetterich equation (21), contributing to the flow of the 4-fermi couplings. Evaluating this term for constant fields and taking the trace over flavor, spinor, and momentum degrees of freedom, we infer

$$
\begin{aligned}
& \frac{1}{2} \operatorname{Tr}\left[\frac{1}{2} \tilde{\partial}_{t}\left(\frac{\Delta \Gamma_{k}^{(2)}}{\Gamma_{k, 0}^{(2)}+R_{k}}\right)^{2}\right]= \\
& \frac{2 \Omega}{N_{\mathrm{f}} \pi^{2}} \int_{0}^{\Lambda} \mathrm{d}|q| \tilde{\partial}_{t} \frac{1}{Z_{k}^{2}\left[1+r\left(q^{2} / k^{2}\right)\right]^{2}}\left[\left(\frac{2 N_{\mathrm{f}}-1}{2 N_{\mathrm{f}}} \tilde{\bar{g}}_{k}^{2}-\frac{3}{2 N_{\mathrm{f}}} \bar{g}_{k} \tilde{\bar{g}}_{k}-\frac{1}{N_{\mathrm{f}}} \bar{g}_{k}^{2}\right)\left(\bar{\psi} \gamma_{45} \psi\right)^{2}+\left(-\frac{1}{2 N_{\mathrm{f}}} \bar{g}_{k} \tilde{\bar{g}}_{k}-\frac{2 N_{\mathrm{f}}+1}{6 N_{\mathrm{f}}} \bar{g}_{k}^{2}\right)\left(\bar{\psi} \gamma_{\mu} \psi\right)^{2}\right],
\end{aligned}
$$

with $\Omega$ being the spacetime volume. The desired beta functions for $\tilde{\bar{g}}_{k}$ and $\bar{g}_{k}$, respectively, are simply given by $2 N_{\mathrm{f}} / \Omega$ times the coefficient of the corresponding 4-fermi term in Eq. (B8). For $Z_{k} \equiv 1$ and by taking the regulator-dependent constant $\ell_{1}^{(\mathrm{F})}$ from Eq. (27) into account, we end up with the flows of the dimensionless couplings $\tilde{g}$ and $g$ (Eq. (24) as displayed in Eqs. (25) and (26).
[1] W. E. Thirring, Annals Phys. 3, 91 (1958).

[2] M. Franz and Z. Tesanovic, Phys. Rev. Lett. 87, 257003 (2001); M. Franz, Z. Tesanovic and O. Vafek, Phys. Rev. B 66, 054535 (2002) |arXiv:cond-mat/0203333].

[3] I. F. Herbut, Phys. Rev. Lett. 88, 047006 (2002) arXiv:cond-mat/0110188]; I. F. Herbut, Phys. Rev. B 66, 094504 (2002) [arXiv:cond-mat/0202491]; I. F. Herbut, Phys. Rev. Lett. 94, 237001 (2005) [arXiv:cond-mat/0410557].

[4] N. E. Mavromatos and J. Papavassiliou, arXiv:cond-mat/0311421

[5] G. W. Semenoff, Phys. Rev. Lett. 53, 2449 (1984).

[6] I. F. Herbut, Phys. Rev. Lett. 97, 146401 (2006) arXiv:cond-mat/0606195].

[7] I. F. Herbut, V. Juricic and B. Roy, Phys. Rev. B 79, 085116 (2009) [arXiv:0811.0610 [cond-mat.str-el]].

[8] J. E. Drut and T. A. Lähde, Phys. Rev. Lett. 102, 026802 (2009) [arXiv:0807.0834 [cond-mat.str-el]]; J. E. Drut, T. A. Lähde and E. Tölö, arXiv:1005.5089 [cond-mat.str-el]; S. Hands and C. Strouthos, Phys. Rev. B 78, 165423 (2008) arXiv:0806.4877 [cond-mat.str-el]]; W. Armour, S. Hands and C. Strouthos, Phys. Rev. B 81, 125105 (2010) [arXiv:0910.5646 [condmat.str-el]]; arXiv:0908.0118 [cond-mat.str-el]; Y. Araki and T. Hatsuda, arXiv:1003.1769 [cond-mat.str-el]; A. Giuliani, V. Mastropietro and M. Porta arXiv:1005.2528 [cond-mat.strel]; W. Wu et al., arXiv:1005.2043 [cond-mat.str-el].

[9] V. P. Gusynin, S. G. Sharapov and J. P. Carbotte, Int. J. Mod. Phys. B 21, 4611 (2007) arXiv:0706.3016 [cond-mat.meshall]].

[10] K. S. Novoselov et al., Science 306, 666 (2004) [arXiv:cond-mat/0410550 [cond-mat.mtrl-sci]].

[11] O. Klein, Z. Phys. 53, 157 (1929); M. I. Katsnelson, K. S. Novoselov and A. K. Geim, Nature Phys. 2, 620 (2006)
[arXiv:cond-mat/0604323 [cond-mat.mes-hall]].

[12] E. Schrödinger, Sitzber. Preuß. Akad. Wiss. Phys.-Math. K1. 24, 418 (1930); M. I. Katsnelson, Eur. Phys. J. B 51, 157 (2006) arXiv:cond-mat/0512337 [cond-mat.mes-hall]]; J. Tworzydlo el al., Phys. Rev. Lett. 96, 246802 (2006) [arXiv:cond-mat/0603315 [cond-mat.mes-hall]].

[13] A. K. Geim and K. S. Novoselov, Nature Mat. 6, 183 (2007) [arXiv:cond-mat/0702595 [cond-mat.mtrl-sci]].

[14] A. H. Castro Neto et al., Rev. Mod. Phys. 81, 109 (2009) arXiv:0709.1163 [cond-mat.other]].

[15] H. Gies, L. Janssen, S. Rechenberger and M. M. Scherer, Phys. Rev. D 81, 025009 (2010) [arXiv:0910.0764 [hep-th]].

[16] J. Alexandre, J. Ellis and N. E. Mavromatos, New J. Phys. 12, 043050 (2010) [arXiv:0901.2532 [hep-th]].

[17] R. D. Pisarski, Phys. Rev. D 29, 2423 (1984).

[18] J. M. Cornwall, Phys. Rev. D 22, 1452 (1980); T. W. Appelquist, M. J. Bowick, D. Karabali and L. C. R. Wijewardhana, Phys. Rev. D 33, 3704 (1986); 33, 3774 (1986); T. Appelquist, D. Nash and L. C. R. Wijewardhana, Phys. Rev. Lett. 60, 2575 (1988); S. Rao and R. Yahalom, Phys. Rev. D 34, 1194 (1986); D. Nash, Phys. Rev. Lett. 62, 3024 (1989).

[19] P. Maris, Phys. Rev. D 54, 4049 (1996) [arXiv:hep-ph/9606214]; T. Appelquist, A. G. Cohen and M. Schmaltz, Phys. Rev. D 60, 045003 (1999) [arXiv:hep-th/9901109].

[20] K. i. Kubota and H. Terao, Prog. Theor. Phys. 105, 809 (2001) [arXiv:hep-ph/0101073]; K. Kaveh and I. F. Herbut, Phys. Rev. B 71, 184519 (2005) |arXiv:cond-mat/0411594].

[21] S. J. Hands, J. B. Kogut and C. G. Strouthos, Nucl. Phys. B 645 , 321 (2002) |arXiv:hep-lat/0208030]; S. J. Hands, J. B. Kogut, L. Scorzato and C. G. Strouthos, Phys. Rev. B 70, 104501 (2004) [arXiv:hep-lat/0404013]. 
[22] V. P. Gusynin and M. Reenders, Phys. Rev. D 68, 025017 (2003) [arXiv:hep-ph/0304302].

[23] C. S. Fischer, R. Alkofer, T. Dahm and P. Maris, Phys. Rev. D 70, 073007 (2004) |arXiv:hep-ph/0407104|; A. Bashir, A. Raya, S. Sanchez-Madrigal and C. D. Roberts, Few Body Syst. 46, 229 (2009) |arXiv:0905.1337 [hep-ph]]; W. Li and G. Z. Liu, Phys. Rev. D 81, 045006 (2010) [arXiv:1003.5299 [cond.mat-str-el]].

[24] G. Parisi, Nucl. Phys. B 100, 368 (1975); S. Hikami and T. Muta, Prog. Theor. Phys. 57, 785 (1977).

[25] S. Hands, Phys. Rev. D 51, $5816 \quad$ (1995) arXiv:hep-th/9411016].

[26] M. Gomes, R. S. Mendes, R. F. Ribeiro and A. J. da Silva, Phys. Rev. D 43, 3516 (1991).

[27] D. K. Hong and S. H. Park, Phys. Rev. D 49, 5507 (1994) |arXiv:hep-th/9307186|.

[28] T. Itoh, Y. Kim, M. Sugiura and K. Yamawaki, Prog. Theor. Phys. 93, 417 (1995) |arXiv:hep-th/9411201]; M. Sugiura, Prog. Theor. Phys. 97, 311 (1997) |arXiv:hep-th/9611198|.

[29] K. i. Kondo, Nucl. Phys. B 450, 251 (1995) [arXiv:hep-th/9502070].

[30] S. Kim and Y. Kim, arXiv:hep-lat/9605021.

[31] S. Christofi, S. Hands and C. Strouthos, Phys. Rev. D 75, 101701 (2007) |arXiv:hep-lat/0701016]; S. Christofi, S. Hands and C. Strouthos, PoS LAT2006, 217 (2006) |arXiv:hep-lat/0703016]; L. Del Debbio and S. J. Hands, Nucl. Phys. B 552, 339 (1999) |arXiv:hep-lat/9902014]; S. Hands and B. Lucini, Phys. Lett. B 461, 263 (1999) [arXiv:hep-lat/9906008].

[32] V. A. Miransky and K. Yamawaki, Phys. Rev. D 55, 5051 (1997) [Erratum-ibid. D 56, 3768 (1997)] [hep-th/9611142]; T. Appelquist, J. Terning and L. C. R. Wijewardhana, Phys. Rev. Lett. 77, 1214 (1996) [hep-ph/9602385]; T. Appelquist, A. Ratnaweera, J. Terning and L. C. R. Wijewardhana, Phys. Rev. D 58, 105017 (1998) |hep-ph/9806472]; M. Harada, M. Kurachi and K. Yamawaki, Phys. Rev. D 68, 076001 (2003) [hep-ph/0305018]; Y. Iwasaki, K. Kanaya, S. Kaya, S. Sakai and T. Yoshie, Phys. Rev. D 69, 014507 (2004) [hep-lat/0309159].

[33] H. Gies and J. Jaeckel, Eur. Phys. J. C 46, 433 (2006) arXiv:hep-ph/0507171].
[34] T. A. Ryttov and F. Sannino, Phys. Rev. D 78, 065001 (2008) [arXiv:0711.3745 [hep-th]]; H. S. Fukano and F. Sannino, arXiv:1005.3340 [hep-ph]; L. Vecchi, arXiv:1004.2063 [hepth]; L. Del Debbio, B. Lucini, A. Patella, C. Pica and A. Rago, arXiv:1004.3206 [hep-lat].

[35] J. Braun and H. Gies, JHEP 1005, 060 (2010) [arXiv:0912.4168 [hep-ph]]; JHEP 0606, 024 (2006) |arXiv:hep-ph/0602226]; Phys. Lett. B 645, 53 (2007) |arXiv:hep-ph/0512085|.

[36] S. Weinberg, in S. W. Hawking, W. Israel: General Relativity (Cambridge Univ. Press, 1979) p. 790-831; arXiv:hep-th/9702027, arXiv:0903.0568 [hep-th].

[37] M. Niedermaier and M. Reuter, Living Rev. Rel. 9, 5 (2006); R. Percacci, arXiv:0709.3851 [hep-th]; O. J. Rosten, arXiv:1003.1366 [hep-th].

[38] C. Wetterich, Phys. Lett. B 301, 90 (1993).

[39] K. Osterwalder and R. Schrader, Commun. Math. Phys. 31, 83 (1973).

[40] C. Wetterich, arXiv:1002.3556 [hep-th]; C. Wetterich, Z. Phys. C 48, 693 (1990).

[41] J. Berges, N. Tetradis and C. Wetterich, Phys. Rept. 363, 223 (2002) |arXiv:hep-ph/0005122; K. Aoki, Int. J. Mod. Phys. B 14, 1249 (2000); J. Polonyi, Central Eur. J. Phys. 1, 1 (2003) |arXiv:hep-th/0110026]; J. M. Pawlowski, Annals Phys. 322, 2831 (2007) [arXiv:hep-th/0512261]; H. Gies, arXiv:hep-ph/0611146, B. Delamotte, arXiv:cond-mat/0702365 H. Sonoda, arXiv:0710.1662 [hep-th].

[42] P. Kopietz, L. Bartosch, and F. Schütz, Introduction to the Functional Renormalization Group (Springer, Berlin, 2010).

[43] D. F. Litim, Phys. Rev. D 64, 105007 (2001) [arXiv:hep-th/0103195].

[44] H. Gies, J. Jaeckel and C. Wetterich, Phys. Rev. D 69, 105008 (2004) [arXiv:hep-ph/0312034].

[45] Y. Nambu and G. Jona-Lasinio, Phys. Rev. 122, 345 (1961); 124, 246 (1961).

[46] H. Gies and C. Wetterich, Phys. Rev. D 65, 065001 (2002) [arXiv:hep-th/0107221]; S. Floerchinger and C. Wetterich, Phys. Lett. B 680, 371 (2009) [arXiv:0905.0915 [hep-th]].

[47] M. Salmhofer, C. Honerkamp, W. Metzner, O. Lauscher, Prog. Theor. Phys. 112, 943 (2004); P. Strack, S. Takei and W. Metzner, Phys. Rev. B 81, 125103 (2010) [arXiv:0905.3894 [condmat.str-el]]. 